\title{
The Effects of Trapa japonica Fructus Protects Dexamethasone- Induced Muscle Atrophy in C2C12 Myotubes
}

\author{
Su Young Shin ${ }^{1}$, Yong Hwang ${ }^{1}$, Jin-Woo Jeong ${ }^{1 *}$ \\ ${ }^{1}$ Nakdonggang National Institute of Biological Resources, 137, Donam 2-gil, Sangju-si, Gyeongsangbuk-do 37242, \\ Republic of Korea
}

\begin{abstract}
Muscle atrophy, known as a sarcopenia, is defined as a loss of muscle mass resulting from a reduction in the muscle fiber area or density due to a decrease in muscle protein synthesis and an increase in protein breakdown. Trapa japonica is a small free-floating plant growing mainly in shallow water or swampy regions, native to Europe, Africa and Asia commonly called as the water chestnut or water nut in South Korea. The fruits of Trapa japonica (FTJ) has been used as a tonic in traditional medicine for thousands of years. Although a great deal of work has been carried out on the therapeutic potential of FTJ, its pharmacological mechanisms of action in muscle diseases actions remain unclear. In the present study, we investigated the potential anti-atrophic effects of FTJ ethanol extracts. The MTT assay did not detect cytotoxic effects of FTJ on C2C12 mouse myoblast cells. Subsequently, the anti-atrophic effects of FTJ on skeletal muscle cells was examined by treating C2C12 myotubes with dexamethasone (DEX; $100 \mu \mathrm{M})$ and/or FTJ $(80 \mu \mathrm{g} / \mathrm{ml})$ for $24 \mathrm{~h}$. Compared with the control, myotube diameter was reduced in DEX-treated cells, whereas FTJ treatment protected against DEX-induced muscle atrophy. MAFbx and MuRF1 protein and mRNA expression levels were detected by western blot analysis and reverse transcription-quantitative polymerase chain reaction, respectively. The results demonstrated that FTJ significantly reduced the expression of atrogin-1/MAFbx and MuRF1. Therefore, data from the present study suggest that FTJ inhibits the expression of atrogin-1/MAFbx and MuRF1 in C2C12 cells, and these characteristics may be of value in the development of anti-atrophy functional foods.
\end{abstract}

Keywords: Trapa japonica, Muscle atrophy, Dexamethasone, Human skeletal muscle cells, Myotubes

\section{Introduction}

Trapa japonica, commonly named the water chestnut or water nut, is an aquatic floating aquatic plant native to Europe, Africa and Asia [1]. It is grown as a food product or medicinal plant in Taiwan, China, and South Korea. It belongs to the family Trapaceae and is planted in shallow ponds, swampy lands, and tanks. T. japonica is a crucial source of bioactive substances that contain large quantities of essential proteins, vitamins and minerals. Pharmacological investigations have indicated that different parts of the water chestnut possess general biological activities, like analgesic, anti-bacterial, antidiabetic, anti-inflammatory, anti-microbial, anti-oxidant, antiulcer, morpho-physiological, nootropic, neuroprotective, immunomodulator, and anti-aging [2-5].

Aging is a multifactorial process that is facilitated by a decline in neuromuscular functions and stress tolerance, which results in tissue degeneration and malfunction, most notably in the skeletal muscles. Age-related muscle atrophy and a reduction in skeletal muscle mass and strength is a condition known as sarcopenia (from the Greek for 'lack of flesh').

Corresponding Author: Jin-Woo Jeong, Animal \& Plant Utilization Team, Nakdonggang National Institute of Biological Resources, Gyeongsangbuk-do, Republic of Korea Received date: December 05, 2019; Accepted date: December 11, 2019; Published date: December 12, 2019.

DOI: https://doi.org/10.31546/IJFSNR.1009 
Sarcopenia leads to muscle weakness and greatly affects physical activity and the quality of life of elderly individuals $[6,7]$; sarcopenia is common in elderly people, and is estimated to occur in $5-13 \%$ of people aged $60-70$ years and in $11-50 \%$ of those $\geq 80$ years [8]. The mechanisms of sarcopenia development are actively being studied and involve both intrinsic and extrinsic factors, including the decline of satellite cell activation, altered hormonal status, contraction-induced injury, cellular vacuolization, autophagy, apoptosis and increased oxidative stress [9].

Mouse $\mathrm{C} 2 \mathrm{C} 12$ skeletal muscle cells are an in vitro model that is widely used to study the factors that regulate muscle growth, proliferation and differentiation [10]. The differentiation of myotubes can be induced by $2 \%$ fetal bovine serum (FBS). Previous studies have demonstrated that $\mathrm{C} 2 \mathrm{C} 12$ myoblasts cultured in a growth-factor-deficient state causes the mononucleated myoblasts to exit the cell cycle, which activates the expression of genes that promote myoblast fusions and the formation of multi-nucleated myotubes [11,12]. During this process, changes in cell shape along with cell fusion occur, and myotube phenotypes can be observed within 5-6 days. In addition, when muscle fiber formation (myogenesis) begins, myogenic transcriptional regulatory factors belonging to the MyoD family, including MyoD, myogenin, Myf4 and Myf5, are activated [13]. Amongst these, myogenin has an important role within the MyoD family as it regulates the differentiation of single nucleated myoblasts into multinucleated myofibers [14].

Glucocorticoids are important in the development of muscle atrophy in humans and animals. In both in vivo and in vitro experiments, muscle atrophy is induced by synthetic glucocorticoids such as dexamethasone (DEX) [15]. In skeletal muscles, DEX causes a reduction in protein synthesis and an increase in protein degradation through the ubiquitinproteasome pathway [16]. The ubiquitin-proteasome pathway has been revealed to mediate the degradation of short-lived proteins and long-lived myofibrillar proteins [17]. This protein-degradation system comprises three enzymatic components: the ubiquitin-activating E1 enzyme, the ubiquitinconjugating E2 enzyme and the ubiquitin-ligating E3 enzyme. E3 ubiquitin ligases serve a crucial role in identifying and targeting proteins for proteasomal degradation [17,18]. A previous study characterized two muscle-specific E3 ubiquitin ligases, muscle RING-finger 1 (MuRF1) and muscle atrophy F-box (MAFbx; also known as atrogin1), as markers of skeletal muscle atrophy. Levels of MuRF1 and atrogin1/MAFbx expression are induced early in the atrophy process, prior to the loss of muscle mass [19]. The expression of atrogin1/MAFbx is controlled by forkhead box $\mathrm{O}$, whereas MuRF1 transcription is controlled by nuclear factor- $\kappa \mathrm{B}$ (NFкB) [20].
The present study investigated the potential protective effects of FTJ on DEX-induced muscle atrophy in C2C12 mouse skeletal muscle cells, based on the efficacy of FTJ muscle contraction and release. The anti-atrophy potential of FTJ was determined by focusing on its effects on the expression of atrogin1/MAFbx and MuRF1.

\section{Materials and Methods \\ Preparation of FTJ Extracts}

The fruits of Trapa japonica (FTJ) were air-dried at room temperature and ground to powder using a mechanical grinder. Approximately $5 \mathrm{~g}$ of the powder was then added to $300 \mathrm{ml} 70 \%$ ethanol and stirred continuously at $100 \mathrm{rpm}$ for $24 \mathrm{~h}$ at room temperature. The resulting extract was filtered and the solvent was removed by rotary vacuum evaporation $(\mathrm{N}-1200 \mathrm{~S}$; EYELA, Tokyo, Japan). The extract was then dissolved in dimethyl sulfoxide (DMSO; SigmaAldrich Chemical Co., St. Louis, MO, USA) to obtain a $25 \mathrm{mg} / \mathrm{ml}$ stock solution and stored at $4^{\circ} \mathrm{C}$. This solution was diluted to the desired concentration with physiological saline prior to use.

\section{Cell culture and MTT assay}

C2C12 mouse skeletal muscle cells were obtained from the American Type Culture Collection (Rockville, MD, USA) and cultured in Dulbecco's modified Eagle's medium (DMEM) supplemented with $10 \%$ fetal bovine serum (FBS) and $1 \%$ penicillinstreptomycin at $37^{\circ} \mathrm{C}$ in a humidified chamber containing 95\% air and 5\% CO2. For the cell viability assay, cells were cultured in the absence and presence of various concentrations of FTJ for $24 \mathrm{~h}$. Measurement of cell viability was determined using the MTT assay, which is based on the conversion of MTT to MTT-formazan by mitochondrial enzymes.

Induction of differentiation.

C2C12 myoblasts were grown to $70-80 \%$ confluence in culture dishes at $37^{\circ} \mathrm{C}$, trypsinized and seeded (3x104 cells/well) into 6-well culture plates for experiments. Cells were grown to $70-80 \%$ confluence in DMEM supplemented with $10 \% \mathrm{FBS}$ at $37^{\circ} \mathrm{C}$ for 24 $\mathrm{h}$, at which time the medium was replaced with DMEM containing 2\% FBS to induce myotube differentiation; the medium was replaced every 2 days. Cells were allowed to differentiate for 6 days, at which point $90 \%$ of the cells had fused into myotubes. 


\section{Treatment with DEX and FTJ}

Following 6 days of differentiation, $\mathrm{C} 2 \mathrm{C} 12$ myotubes were treated with 10,50 or $100 \mu \mathrm{M}$ DEX at $37^{\circ} \mathrm{C}$ for $24 \mathrm{~h}$ for concentration screening. The $\mathrm{C} 2 \mathrm{C} 12$ myotubes were subdivided into four groups: i) the control group, in which cells were incubated in serum free medium (SFM; DMEM containing $100 \mathrm{U} / \mathrm{ml}$ penicillin and $100 \mathrm{mg} / \mathrm{ml}$ streptomycin); ii) the DEX group, in which cells were treated with $100 \mu \mathrm{M}$ DEX; iii) the DEX+FTJ group, in which cells were treated with $100 \mu \mathrm{M}$ DEX and $80 \mu \mathrm{g} / \mathrm{ml} \mathrm{FTJ}$; and iv) the FTJ group, in which cells were treated with $80 \mu \mathrm{g} / \mathrm{ml}$ FTJ. All groups were incubated at $37^{\circ} \mathrm{C}$ for $24 \mathrm{~h}$, prior to harvesting cells for experiments.

\section{Measurement of myotube diameter}

Images of myotube cultures were captured with a phase contrast microscope set to x100 magnification at 2, 4, 5 and 6 days following induction of differentiation. Images of myotube cultures were also captured following treatment with DEX $(100 \mu \mathrm{M})$ and/or FTJ $(80 \mu \mathrm{g} / \mathrm{ml})$ for $24 \mathrm{~h}$.
A total of 50 myotube diameters from at least 10 random fields were measured using ImageJ software (version 4.16; National Institutes of Health, Bethesda, MD, USA).

\section{Protein extraction and western blot analysis}

Cells were harvested and lysed with lysis buffer $(20$ $\mathrm{mM}$ sucrose, $1 \mathrm{mM}$ EDTA, $20 \mu \mathrm{M}$ Tris- $\mathrm{HCl}, \mathrm{pH} 7.2,1$ $\mathrm{mM}$ DTT, $10 \mathrm{mM} \mathrm{KCl}, 1.5 \mathrm{mM} \mathrm{MgCl} 2$ and $5 \mu \mathrm{g} / \mathrm{ml}$ aprotinin) for $30 \mathrm{~min}$. The protein concentration was measured using a Bio-Rad protein assay (Bio-Rad, Hercules, CA, USA). For western blot analysis, an equal quantity of protein was subjected to electrophoresis on sodium dodecyl sulfate (SDS)polyacrylamide gel and transferred to a nitrocellulose membrane (Schleicher \& Schuell, Inc., Keene, NH, USA). Blots were probed with the desired antibodies for $1 \mathrm{~h}$, incubated with the diluted enzyme-linked secondary antibody and visualized by enhanced chemiluminescence according to the manufacturer's instructions (Amersham Pharmacia Biotech).

Table 1: Primary antibodies used in western blot analysis

\begin{tabular}{cccc}
\hline \hline Antibody & Dilution & Product no. & Species of origin and supplier \\
\hline \hline Anti-Myogenin & $1: 1,000$ & SC-12732 & Mouse monoclonal, Santa Cruz Biotechnology, Inc. \\
Anti-MuRF1 & $1: 1,000$ & SC-27642 & Goat polyclonal, Santa Cruz Biotechnology, Inc. \\
Anti-atrogin1/MAFbx & $1: 1,000$ & SC-27645 & Goat polyclonal, Santa Cruz Biotechnology, Inc. \\
Anti-actin & $1: 1,000$ & SC-8432 & Mouse monoclonal, Santa Cruz Biotechnology, Inc. \\
\hline \hline
\end{tabular}

MAFbx, muscle atrophy F-box; MuRF1, muscle RING finger 1.

Table 2: Secondary antibodies used in western blot analysis

\begin{tabular}{ccc}
\hline \hline Antibody & Dilution & Product no. \\
\hline \hline Goat anti-mouse IgG & $1: 10,000$ & SC-2031 \\
Rabbit anti-goat IgG & $1: 10,000$ & SC-2768 \\
\hline \hline
\end{tabular}

\section{Reverse transcription-polymerase chain reaction (RT-} PCR)

Total RNA was isolated using TRIzol reagent (Invitrogen, CA). Total RNA (1 $\mu \mathrm{g})$ obtained from cells was primed with random hexamers to synthesize complementary DNA using MMLV reverse transcriptase (Promega, Madison, WI) according to the manufacturer's instructions.
Polymerase chain reaction (PCR) was performed for amplification of the MuRF1 and atrogin1/MAFbx genes from the cDNA and glyceraldehyde-3-phosphate dehydrogenase (GAPDH) was used as an internal control.

Oligonucleotide primers for MuRF1, atrogin1/MAFbx and GAPDH are shown in Table 3. RT-PCR reactions were incubated for an initial denaturation at $95^{\circ} \mathrm{C}$ for $10 \mathrm{~min}$, followed by 40 cycles of: $95^{\circ} \mathrm{C}$ for $15 \mathrm{sec}$, $55^{\circ} \mathrm{C}$ for $15 \mathrm{sec}$, and $72^{\circ} \mathrm{C}$ for $15 \mathrm{sec}$. Amplification products obtained by PCR were electrophoretically separated on $1 \%$ agarose gel and visualized by $\mathrm{EtBr}$ staining. 
Table 3: Oligonucleotide primer sequences used in RT-PCR

\begin{tabular}{cc}
\hline \hline Gene & Sequence \\
\hline \hline \multirow{2}{*}{ MuRF1 } & F: 5'-TGT CTG GAG GTC GTT TCC G-3' \\
& R: 5'-GTG CCG GTC CAT GAT CAC TT-3' \\
MAFbx & F: 5'-ATG CAC ACT GGT GCA GAG AG-3' \\
& R: 5'-TGT AAG CAC ACA GGC AGG TC-3' \\
GAPDH & F: 5'-CGG AGT CAA CGG ATT TGG TCG TAT-3' \\
& R: 5'-AGC CTT CTC CAT GGT GGT GAA GAC-3' \\
\hline \hline
\end{tabular}

\section{Statistical analysis}

The data are expressed as the mean \pm SD. A statistical comparison was performed using one-way analysis of variance followed by Fisher's exact test. The significant differences between the groups were determined using unpaired Student's t-test. $\mathrm{p}<0.05$ was considered to indicate a statistically significant difference.

A)

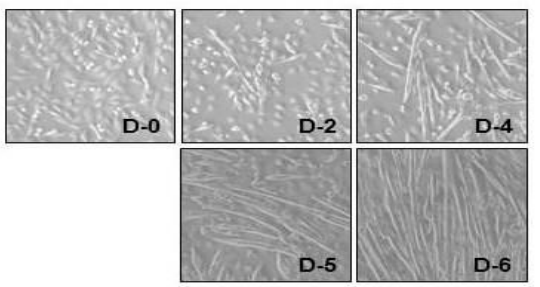

c)

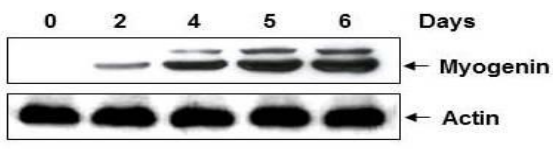

\section{Results}

\section{Confirmation of C2C12 myotube differentiation}

$\mathrm{C} 2 \mathrm{C} 12$ skeletal muscle myoblasts were induced to differentiate in a mitogen poor media such as $2 \%$ FBS, fusing to form multinucleate myotubes [21]. Fig. 1 illustrates that the cell diameter remained similar following 2, 4, 5 and 6 days of differentiation. In addition, the level of myogenin expression, a factor that regulates terminal differentiation of muscle cells, was similar following 2, 4 and 5 days then increased following 6 days. These results indicate complete differentiation of myoblasts into myotubes at this time (6 days).

B)

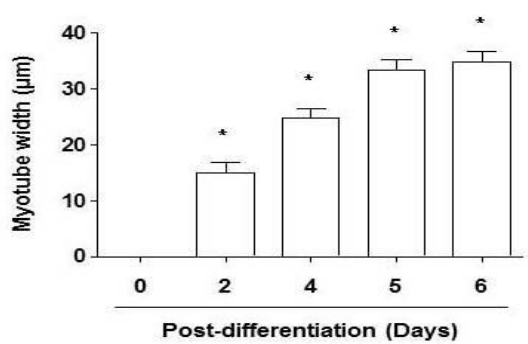

D)

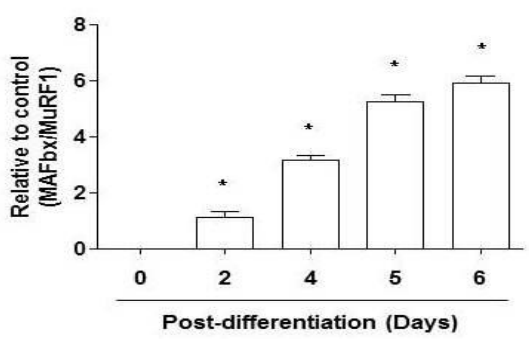

Figure 1: Differentiation of $\mathrm{C} 2 \mathrm{C} 12$ myoblasts into myotubes. (A) Representative images of myoblasts prior to induction and on days 2, 4, 5 and 6 following incubation with $2 \%$ fetal bovine serum. Magnification, x100. (B) Myotube diameters and (C and D) myogenin protein expression levels were compared across the differentiation stages. Results are presented as the mean \pm standard deviation of three independent experiments. ${ }^{*} \mathrm{p}<0.05$ vs. corresponding control group (D 0). CON, control; D, day. 


\section{DEX induced muscle atrophy in $\mathrm{C2C12}$ myotubes}

MuRF1 and atrogin1/MAFbx protein expression levels were measured in C2C12 myotubes treated with DEX (10, 50 or 100 $\mu \mathrm{M})$ for $24 \mathrm{~h}$ (Fig. 2A and B). Treatment with DEX led to increased protein expression levels for MuRF1 and atrogin1/MAFbx, and the levels of expression increased in a dose dependent manner. These data suggest that DEX may induce muscle atrophy in $\mathrm{C} 2 \mathrm{C} 12$ myotubes. In addition, as shown in Fig. 2C, when compared with the control group, the C2C12 myotube was markedly atrophied in the $100 \mu \mathrm{M}$ DEX treated group. Therefore, all further experiments were performed following treatment with $100 \mu \mathrm{M} \mathrm{DEX}$.

\section{Toxicity of FTJ in C2C12 myoblast}

The MTT assay was used to determine FTJ cytotoxicity to C2C12 myoblasts, which demonstrated no cytotoxic effects following FTJ treatment for $24 \mathrm{~h}$ (Fig. 3). Therefore, all further experiments were performed $24 \mathrm{~h}$ following treatment with FTJ $(80 \mu \mathrm{g} / \mathrm{ml})$, which is the optimum concentration without toxicity as described by Choi et al. [22].

A)

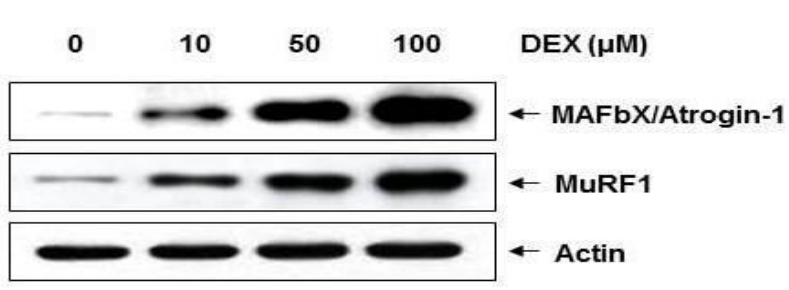

C)

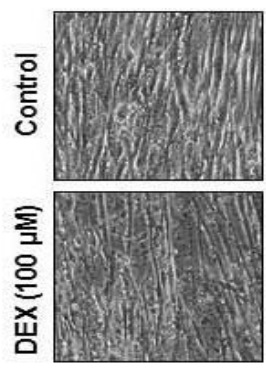

Inhibitory effect of FTJ on DEX induced myotube atrophy

C2C12 myotubes were allowed to differentiate for 6 days, followed by DEX $(100 \mu \mathrm{M})$ and/or FTJ (80 $\mu \mathrm{g} / \mathrm{ml}$ ) treatment for a further $24 \mathrm{~h}$. As a result, when compared with the control group, the DEX treated group exhibited a $52 \%$ reduction in cell diameter, whereas the FTJ treated group exhibited a 5\% increase in cell diameter. In addition, DEX induced reduction of cell diameter was suppressed by co treatment with FTJ (Fig. 4A and B). The anti-atrophic effects of FTJ were investigated by examining the protein and mRNA expression levels of the muscle atrophy markers MuRF1 and atrogin1/MAFbx in C2C12 myotubes that were treated with DEX $(100 \mu \mathrm{M})$ and FTJ $(80 \mu \mathrm{g} / \mathrm{ml})$ for $24 \mathrm{~h}$ (Fig. $5 \mathrm{~A}$ and B). Cells treated with DEX alone exhibited a marked increase in the mRNA and protein expression levels of MuRF1 and atrogin1/MAFbx compared with cells in the untreated control group (Fig. $5 \mathrm{~A}$ and B). The DEX induced upregulation of MuRF1 and atrogin1/MAFbx was suppressed by co treatment with FTJ $(80 \mu \mathrm{g} / \mathrm{ml})$. Therefore, FTJ may be able to inhibit atrophy and induce hypertrophy pathways in $\mathrm{C} 2 \mathrm{C} 12$ myotubes.

B)

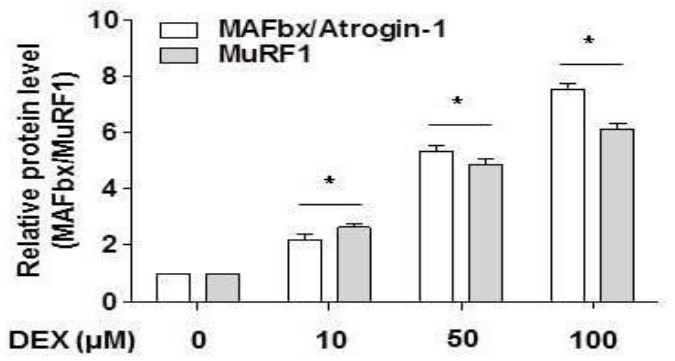

Figure 2: Effects on MuRF1 and MAFbx protein expression in $\mathrm{C} 2 \mathrm{C} 12$ myoblasts treated with various concentrations of DEX. (A) Western blot and (B) densiᄀtometric analysis of MuRF1 and atrogin1/MAFbx protein expression levels following treatment with 10, 50 and $100 \mu \mathrm{M} \mathrm{DEX}$. Results are expressed as the mean \pm standard deviation of three independent experiments. ${ }^{*} \mathrm{p}<0.05 \mathrm{vs}$. corresponding control group. (C) Cells were treated with $100 \mu \mathrm{M}$ DEX and cell morphology was analyzed (original magnification, x100). CON,control; DEX, dexamethasone; MAFbx, muscle atrophy F box; MuRF1, muscle RING finger 1. 


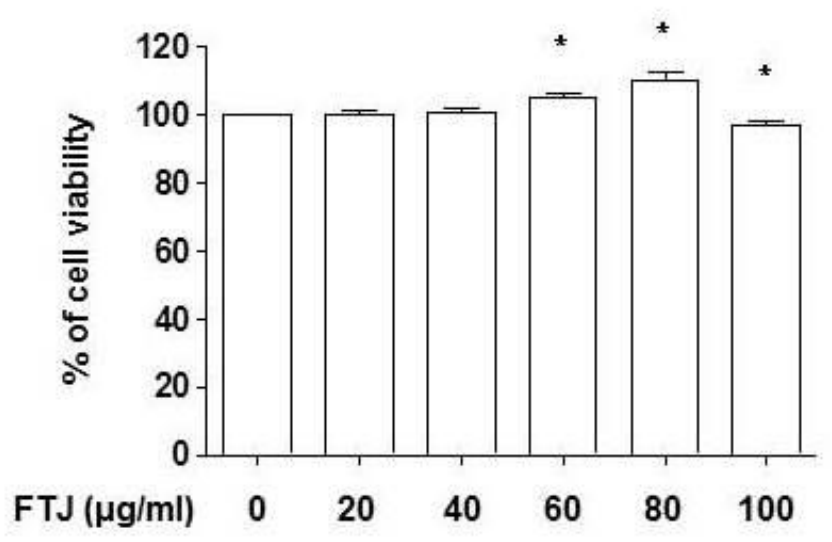

Figure 3: Effects of FTJ on C2C12 myotube viability. Cells (3x104 cells/well) were seeded in 6 well plates in DMEM supplemented with $10 \%$ fetal bovine serum. Following $24 \mathrm{~h}$ incubation, cells were serum starved for $4 \mathrm{~h}$ and treated with FTJ at the indicated concen 7 trations for $24 \mathrm{~h}$. Results are presented as the mean \pm standard deviation of three independent experiments. ${ }^{*} \mathrm{p}<0.05$ vs. corresponding control group.

A)

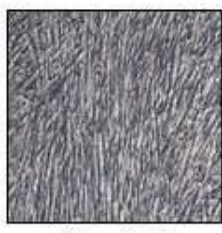

Control

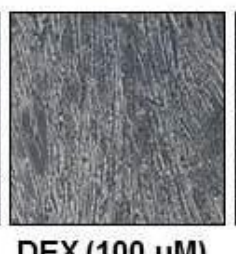

$\operatorname{DEX}(100 \mu \mathrm{M})$

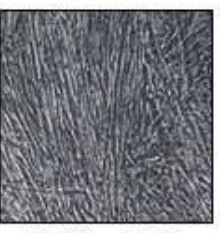

$\mathrm{DEX}+\mathrm{FTJ} \quad \mathrm{FTJ}(80 \mu \mathrm{g} / \mathrm{ml})$

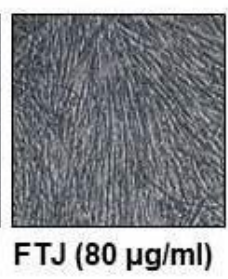

B)

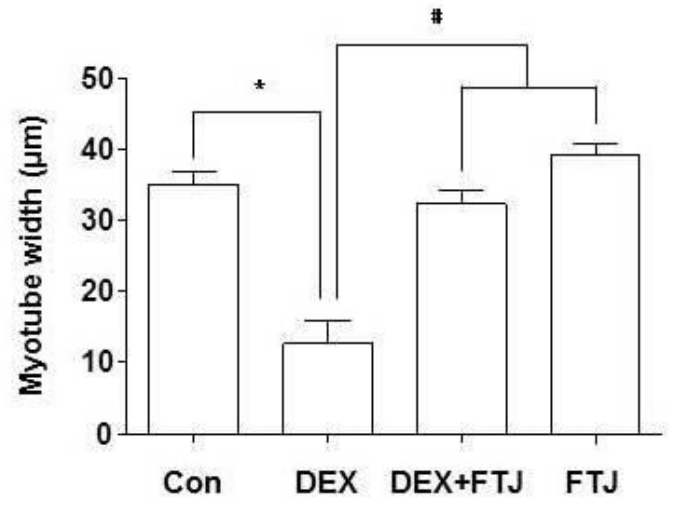

Figure 4: Effects of FTJ and/or DEX on differentiated C2C12 myotubes. (A) Representative images of C2C12 myotubes following the various DEX and FTJ treatments. (B) Comparison of myotube diameters among the four groups. Results are presented as the mean \pm standard deviation of three independent experiments. ${ }^{*} \mathrm{p}<0.05$ vs. corresponding control group; $\#$ p $<0.05$ vs. corresponding DEX only treatment group. CON, control; DEX, dexamethasone; FTJ, fruits of Trapa japonica. 
A)

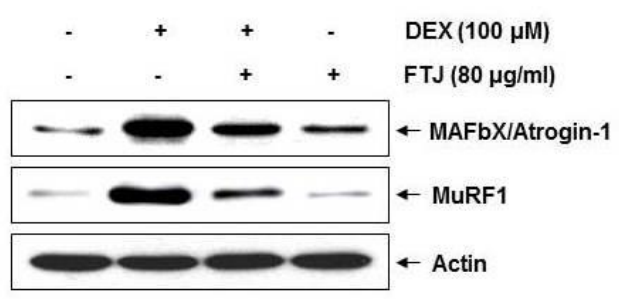

C)

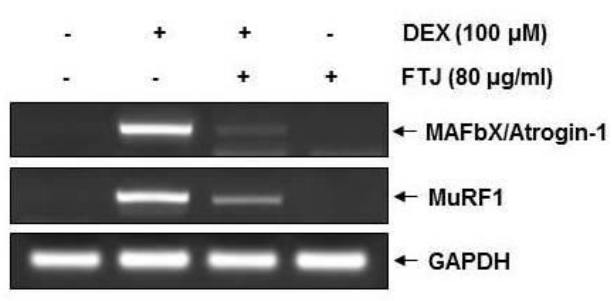

B)

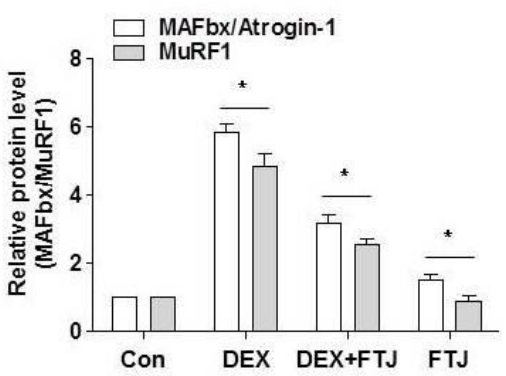

D)

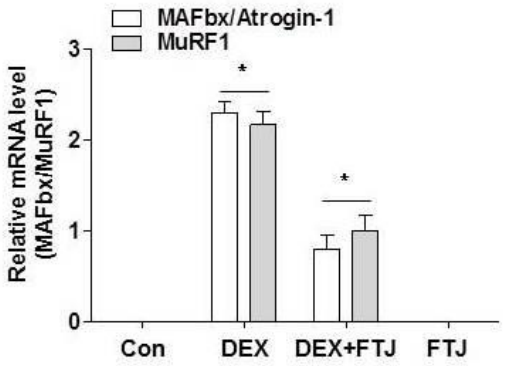

Figure 5: Inhibition of MuRF1 and atrogin1/MAFbx expression in C2C12 myotubes treated with DEX (100 $\mu \mathrm{M})$ and/or FTJ $(80 \mu \mathrm{g} / \mathrm{ml})$ for $24 \mathrm{~h}$. MuRF1 an atrogin1/MAFbx Protein expression levels were analyzed by (A) western blot and (B) densitometric analysis; Actin was used to normalize expression. (C) mRNA expression levels were quantified by reverse transcription polymerase chain reaction and (D) densitometric analysis; GAPDH was used to normalize expression. Results are presented as the mean \pm stan $\neg$ dard deviation of three independent experiments. ${ }^{*} \mathrm{p}<0.05$ vs. corresponding control group; $\#$ p $<0.05$ vs. corresponding only DEX treatment group. CON, control; DEX, dexamethasone; MAFbx, muscle atrophy F box; MuRF1, muscle RING finger 1 FTJ, fruits of Trapa japonica.

\section{Discussion}

Skeletal muscle atrophy refers to the decline in muscle mass and strength that may occur as a result of various conditions, including denervation, injury, glucocorticoid treatment, starvation, cancer, joint immobilization, sepsis and aging [23]. The present study focused on the progression of muscle atrophy caused by aging.

In the present study, anti-atrophic effects of the fruits of Trapa japonica (FTJ) were investigated in $\mathrm{C} 2 \mathrm{C} 12$ myotubes. $\mathrm{C} 2 \mathrm{C} 12$ mouse skeletal muscle cells are commonly used as a model for myotube differentiation to examine the signaling pathways involved in muscle atrophy. The effects of the FTJ on the muscle atrophy in $\mathrm{C} 2 \mathrm{C} 12$ myotubes were determined by first culturing $\mathrm{C} 2 \mathrm{C} 12$ myoblasts in media containing 2\% FBS for 6 days to induce differentiation. Following differentiation, muscle atrophy was induced by treating the cells with DEX. DEX treatment has been previously demonstrated to reduce muscle mass, largely owing to the breakdown of muscle proteins due to upregulated catabolism by the ubiquitin prote $\neg$ asome system [24,25]. Administration of high concentrations of DEX has been revealed to cause muscle atrophy in animals and humans [26].
Results from the present study demonstrated that C2C12 myotubes respond to increasing concentrations of DEX $(10,50$ and $100 \mu \mathrm{M})$ in a dose dependent manner, as revealed by the increased expression of MuRF1 and atrogin1/MAFbx protein levels. Prior to determining the protective effects of FTJ on muscle atrophy, the potential toxicity of FTJ on $\mathrm{C} 2 \mathrm{C} 12$ myoblasts was determined using an MTT assay, which revealed that FTJ treatment had no cytotoxic effects on C2C12 myoblasts. We next assessed the protective effects of FTJ by measuring myotube diameter. Compared with the control group, the DEX treated group exhibited a $52 \%$ reduction in cell diameter, whereas the FTJ treated group exhibited a 5\% increase in cell diameter. Subsequently, the effects of FTJ on $\mathrm{C} 2 \mathrm{C} 12$ myotube atrophy were investigated by examining the expression levels of muscle atrophy markers MuRF1 and atrogin1/MAFbx, as they are upregulated in a number of catabolic conditions [27]. Glucocorticoids such as DEX have been demonstrated to promote myosin heavy chain degradation through the activation of the E3 ligase MuRF1 [28]. 
Therefore, the downregulation of MuRF1 and atrogin1/MAFbx expression may inhibit muscle atrophy. Western blot and RT PCR analyses in the present study revealed that FTJ treatment led to a decrease in the mRNA and protein expression levels of MuRF1 and atrogin1/MAFbx, suggesting that FTJ may potentially be used in anti-atrophy functional foods.

In conclusion, the present study provides important new information about the influence of FTJ on DEX induced muscle atrophy. The results provide molecular evidence that the anti-atrophic effects of FTJ are due to the downregulated expression of the muscle specific E3 ubiquitin ligases MuRF1 and atrogin1/MAFbx. Future studies on the anti-atrophic effects of FTJ are required, and should involve identifying the signaling pathways that may be associated with the antiatrophic effects, such as insulin like growth factor 1/Akt and myostatin.

\section{Conflict of interest}

The authors declare that there are no conflicts of interest.

\section{Acknowledgments}

This research was supported by a grant from the Nakdonggang National Institute of Biological Resources (NNIBR), funded by the Ministry of Environment (MOE) of the Republic of Korea (NNIBR201902104), and the Basic Science Research Program through the National Research Foundation of Korea (NRF) Grant funded by the Korea government (NRF2016R1C1B1014724).

\section{References}

1. Takano A, Kadono Y. Allozyme variations and classification of Trapa (Trapaceae) in Japan. Aquat. Bot. 2005;83:108-118.

2. Lee D, Lee OH, Choi G, Kim JD. Antioxidant and AntiAdipogenic Activities of Trapa japonica Shell Extract Cultivated in Korea. Prev. Prev Nutr Food Sci. 2017; 22:327-334.

3. Kim YS, Hwang JW, Jang JH, et al. Trapa japonica Pericarp Extract Reduces LPS-Induced Inflammation in Macrophages and Acute Lung Injury in Mice. Molecules. 2016; 21:392.

4. Kim YS, Kim EK, Hwang JW, et al. Characterization of the antioxidant fraction of Trapa japonica pericarp and its hepatic protective effects in vitro and in vivo. Food Funct. 2016; 7:1689-1699.

5. Kim YS, Hwang JW, Han YK, et al. Antioxidant activity and protective effects of Trapa japonica pericarp extracts against tert-butylhydroperoxide-induced oxidative damage in Chang cells. Food Chem Toxicol. 2014; 64:49-56.
6. Wenz T, Rossi SG, Rotundo RL, et al. Increased muscle PGC 1alpha expression protects from sarcopenia and metabolic disease during aging. Proc Natl Acad Sci U S A. 2009; 106:2040520410.

7. Doherty TJ. Invited Review: Aging and sarcopenia. J Appl Physiol (1985). 2003; 95:17171727.

8. Marzetti E and Leeuwenburgh C. Skeletal muscle apoptosis, sarcopenia and frailty at old age. Exp Gerontol. 2006; 41:1234-1238.

9. Dupont Versteegden EE. Apoptosis in muscle atrophy: Relevance to sarcopenia. Exp Gerontol. 2005; 40:473-481.

10. Artaza JN, Bhasin S, Mallidis C, et al. Endogenous expression and localization of myostatin and its relation to myosin heavy chain distribution in $\mathrm{C} 2 \mathrm{C} 12$ skeletal muscle cells. J Cell Physiol. 2002; 190:170-179.

11. Aoki MS, Miyabara EH, Soares AG, et al. mTOR pathway inhibition attenuates skeletal muscle growth induced by stretching. Cell Tissue Res. 2006; 324:149-156.

12. Weintraub H. The MyoD family and myogenesis: Redundancy, networks, and thresholds. Cell. 1993; 75:1241-1244.

13. Melo F, Carey DJ, Brandan E. Extracellular matrix is required for skeletal muscle differentiation but not myogenin expression. J Cell Biochem. 1996; 62:227-39.

14. te Pas MF, Soumillion A, Harders FL, et al. Influences of myogenin genotypes on birth weight, growth rate, carcass weight, backfat thickness, and lean weight of pigs. J Anim Sci. 1999; 77:23522356.

15. Sandri M, Sandri C, Gilbert A, et al. Foxo transcription factors induce the atrophy related ubiquitin ligase atrogin 1 and cause skeletal muscle atrophy. Cell. 2004; 117:399-412.

16. Zhao W, Qin W, Pan J, et al. Dependence of dexamethasone induced Akt/FOXO1 signaling, upregulation of MAFbx, and protein catabolism upon the glucocorticoid receptor. Biochem Biophys Res Commun. 2009; 378:668-672.

17. Glass DJ. Skeletal muscle hypertrophy and atrophy signaling pathways. Int $\mathrm{J}$ Biochem Cell Biol. 2005; 37:1974-1984.

18. Hershko A, Ciechanover A. The ubiquitin system. Annu Rev Biochem. 1998; 67:425-479. 
19. Lecker SH, Jagoe RT, Gilbert A, et al. Multiple types of skeletal muscle atrophy involve a common program of changes in gene expression. FASEB J. 2004; 18: 39-51.

20. Meng SJ, Yu LJ. Oxidative stress, molecular inflammation and sarcopenia. Int J Mol Sci. 2010; 11: 1509-1526.

21. Cooper ST, Maxwell AL, Kizana E, et al. C2C12 co culture on a fibroblast substratum enables sustained survival of contractile, highly differentiated myotubes with peripheral nuclei and adult fast myosin expression. Cell Motil Cytoskeleton. 2004; 58:200-211.

22. Choi YH, Yamaguchi K, Oda T, Nam TJ. Chemical and mass spectrometry characterization of the red alga Pyropia yezoensis chemoprotective protein (PYP): Protective activity of the $\mathrm{N}$ terminal fragment of PYP1 against acetaminophen induced cell death in Chang liver cells. Int J Mol Med. 2015; 35:271-276.

23. Gomes MD, Lecker SH, Jagoe RT, et al. Atrogin1, a muscle specific $\mathrm{F}$ box protein highly expressed during muscle atrophy. Proc Natl Acad Sci U S A. 2001; 98:14440-14445.

24. Auclair D, Garrel DR, Chaouki ZA, Ferland LH. Activation of the ubiquitin pathway in rat skeletal muscle by cata-bolic doses of glucocorticoids. Am J Physiol. 1997; 272:C1007-C1016.

25. Jackman RW, Kandarian SC. The molecular basis of skeletal muscle atrophy. Am J Physiol Cell Physiol. 2004; 287:C834-C843.

26. Mitch WE, Goldberg AL. Mechanisms of muscle wasting. The role of the ubiquitin proteasome pathway. N Engl J Med. 1996; 335:1897-1905.

27. Krawiec BJ, Nystrom GJ, Frost RA, et al. AMP activated protein kinase agonists increase mRNA content of the muscle specific ubiquitin ligases MAFbx and MuRF1 in C2C12 cell. Am J Physiol Endocrinol Metab. 2007 Jun;292(6):E1555-E1567.

28. Clarke BA, Drujan D, Willis MS, et al. The E3 ligase MuRF1 degrades myosin heavy chain protein in dexamethasone treated skeletal muscle. Cell Metab. 2007; 6:376-385. 